Classification

Physics Abstracts

$42.30-81.05-81.40$

\title{
Image Analysis of Austenite and Carbides Coarsening in a Fe-Mo-C Steel
}

\author{
Jean-Marc Chaix, Pei Hong Yang, Michel Ardin and Madeleine Durand-Charre \\ Laboratoire de Thermodynamique et de Physico-Chimie Métallurgiques, \\ UMR 5614 CNRS-INPG/UJF, ENSEEG, BP 75, 38402 Saint Martin d'Hères, France
}

\begin{abstract}
Résumé. - Trois populations significatives d'objets évoluent au cours du recuit de l'acier Fe-Mo-C étudié: les grains d'austénite, des carbures $\mathrm{M}_{6} \mathrm{C}$ inter et intra-granulaires. Ces trois populations sont extraites à partir de transformations de morphologie mathématique. Des mesures de diamètre équivalent peuvent ensuite être réalisées pour analyser les mécanismes d'évolution par comparaison avec des modèles.
\end{abstract}

\begin{abstract}
Three classes of objects evolve inside the present Fe-Mo-C steel during annealing: austenite grains, intra and inter-granular $\mathrm{M}_{6} \mathrm{C}$ carbides. These populations are separated using transformations based on mathematical morphology. Size measurements (equivalent diameter) enable the analysis of the mechanisms through comparison with growth models.
\end{abstract}

\section{Introduction}

This work is part of a study which aims to analyse coarsening phenomena in a Fe-12.4wt\%Mo$1.3 \mathrm{wt} \% \mathrm{C}$ alloy, model of high speed steel. This alloy was annealed in a temperature range in which it forms $\mathrm{M}_{6} \mathrm{C}$, austenite $(\gamma)$ and liquid [1]. A typical micrograph of quenched samples is presented in Figure 1. The dendritic pattern evolved into a set of globular shaped $\gamma$-phase objects, referred as "dendrites" in the following. These "dendrites" coarsened inside the inter-dendritic liquid phase, together with the large inter-dendritic carbides. The intra-dendritic carbides, inside the $\gamma$ dendrites, are either present at the beginning of the experiment or trapped during the dendrite coarsening process. These three populations of particles are involved in Ostwald ripening processes at the experiment temperature. The fine eutectic carbides surrounding the dendrites in Figure 1 have been formed during quenching from the liquid phase and have not to be considered. The present paper describes the methods developped to discriminate the objects of interest and to characterize their size distribution and mean growth.

\section{Extraction of $\gamma$ Dendrites}

The thresholded image of the $\gamma$ phase (white in Fig. 2a) must be transformed to get the $\gamma$ dendrites. The image treatment is based on the analysis of the morphological characteristics of the image. Intra-dendritic carbides are small isolated objects which must be removed. The inter-dendritic 


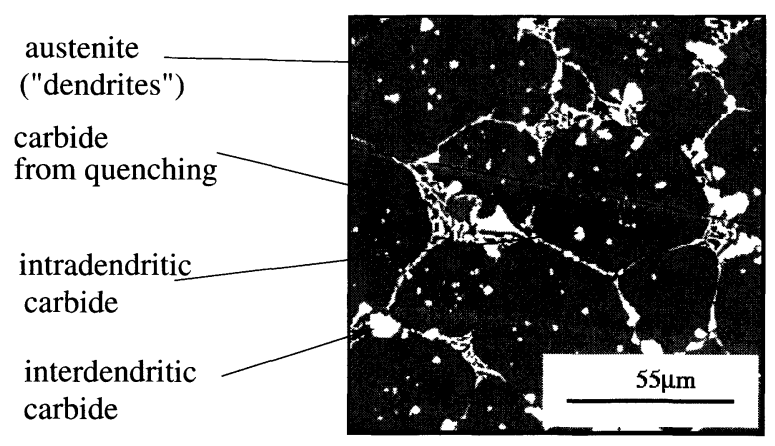

Fig. 1. - Backscattered electron micrograph of the quenched alloy $(420 \times 420$ pixels image cut from the original $512 \times 512$ image at mag. 1000$)$.

zone is marked by the carbides which constitute a discontinuous network with narrow and large parts separated by small $\gamma$ parts formed from the liquid on quenching: it has to be connected and filled. These observations provide the guideline of the chosen treatment, based on classic mathematical morphology transformations [2].

The first step consists in removing the smaller intra-dendritic carbides. The inter-dendritic network is reinforced by a small closing of the carbide image (Fig. 2b): this connects the network but prevents connecting the intra-dendritic carbides. The smaller of these latter carbides are easily removed by skeletonizing (Fig. 2c) and clipping (Fig. 2d), then finally reconstructing using the clipped skeleton as seed and the original image as template (Fig. 2e). Some of the larger intradendritic carbide grains are still present: the same sequence with larger transformation sizes is therefore performed (Figs. $2 \mathrm{f}$ to i). This two-step procedure is necessary, as a large closing in the first step would link many small objects and create clipping-unremovable closed skeleton parts. The dendrite image (Fig. 2i), rebuilt with (Fig. 2f) as template, is finally separated into objects, with the classic binary watershed algorithm (ultimate erosions).

The closing and clipping sizes have to be adjusted interactively. The intermediate image is therefore presented to the user after each structuring element size dependent treatment, which is repeated with different values if not satisfactory. In samples treated above the eutectic temperature, only the carbides from quenching are present, so that the treatment is simplified.

\section{Extraction of Intra- and Inter-Dendritic Carbides}

The procedure for extracting the intra- and inter-dendritic carbides (Fig. 3) is similar. It is based on the same above observations. In addition, the small objects are more accurately defined than in the previous case, since the magnification is larger: the carbides solidified during quenching are lamellar or fish-bone shaped objects, and their local width is far smaller than the one of the large inter-dendritic carbides.

The first step is a simple $3 \times 3$ pixels conditional opening (Fig. $3 a \rightarrow$ Fig. $3 b$ ). Some defects, such as the polishing groove in Figures $3 \mathrm{a}$ and 1 are removed, as well as unsignificant small carbides. The second step involves, as previously, a closing followed by a skeleton transformation and a clipping (Figs. 3c, d). The clipping size is chosen to eliminate the intra-dendritic parts. The interdendritic zone is obtained from the clipped skeleton, the intra-dendritic objects (Fig. 3e) being the carbides which have no contact with the inter-dendritic zone.

To separate the large inter-dendritic objects from the ones from quenching, an automatic watershed separation is performed, which breaks the quenching carbides into very small parts, easily removed by a conditional opening. The inter-dendritic carbides are therefore readily obtained 
a)

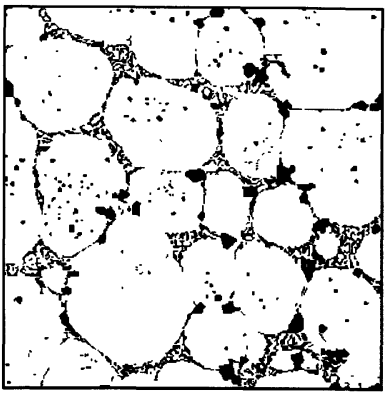

d)

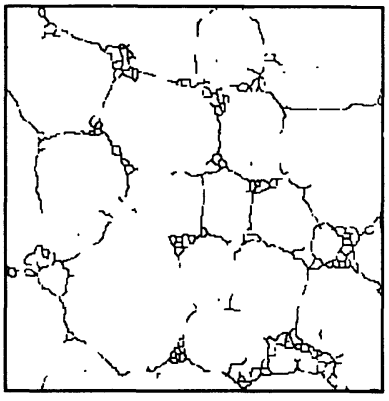

g)

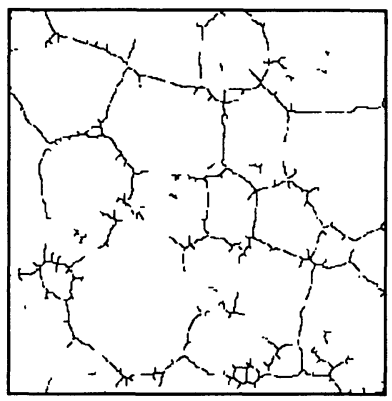

b)

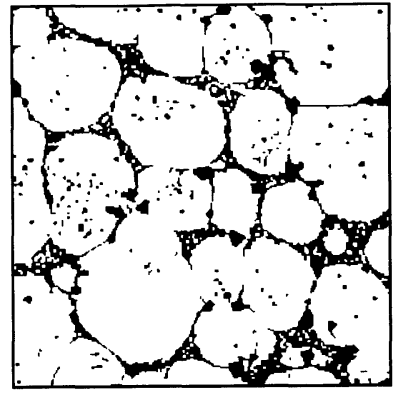

e)

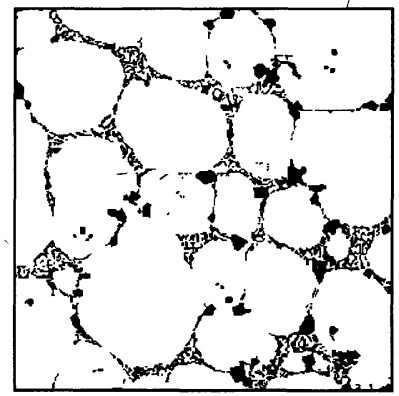

h)

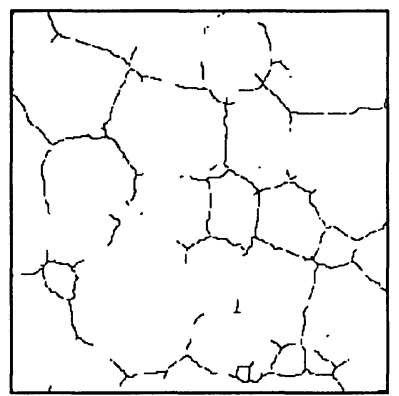

i)

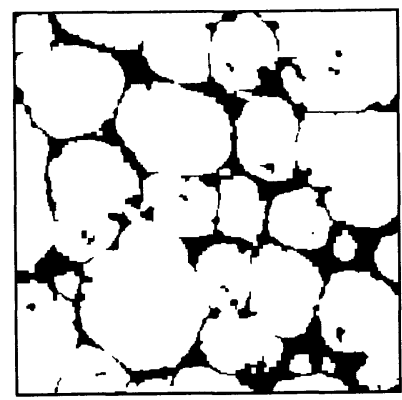

f)

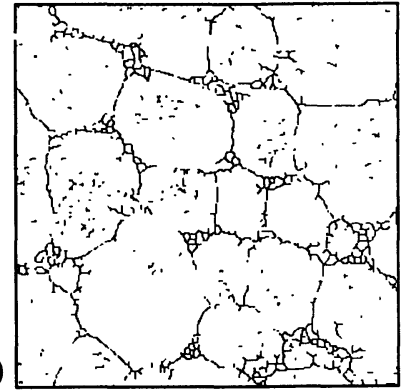

c)

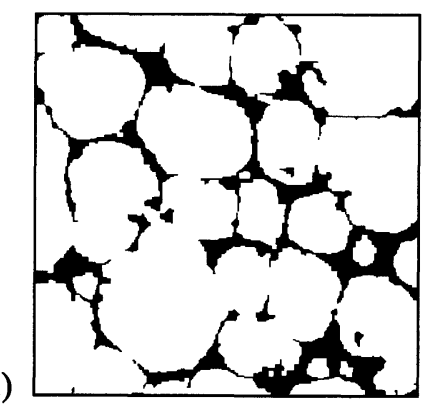

j)

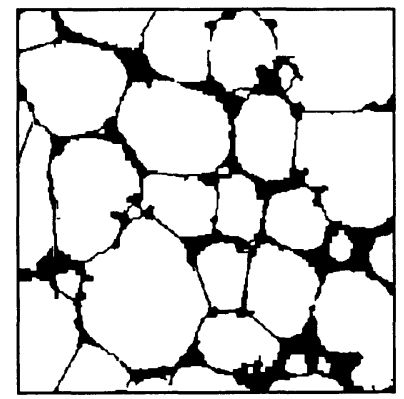

Fig. 2. - Main steps of the extraction of "dendrites": a) original binary image, with $\gamma$ phase in white and carbide phase in white; b) after small size closing of the carbide phase; c) skeleton of the closed carbide phase; d) small size clipped skeleton e) reconstructed inter+large intra-dendritic carbides; f) medium size closing of $d ; g$ ) skeleton; $h$ ) medium size clipped skeleton; i) reconstructed f from $h$; j) separated dendrites after watershed on i. $(300 \times 300$ pixels cut from $512 \times 512$ images at mag. 500$)$. 
a)

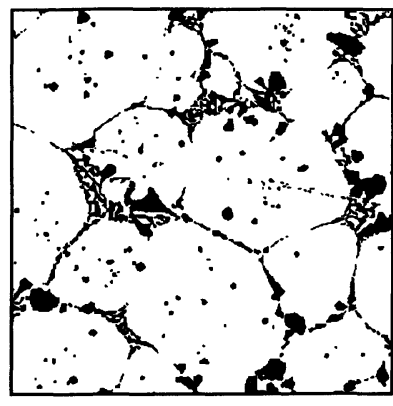

d)

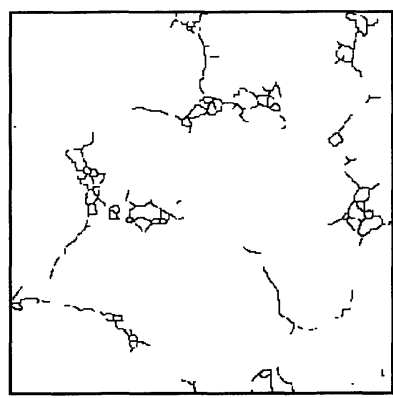

b)

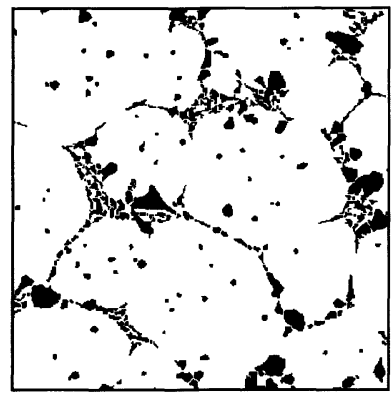

e)

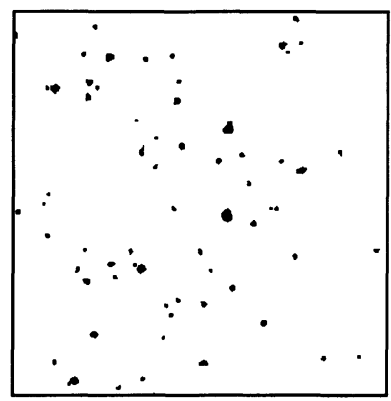

c)

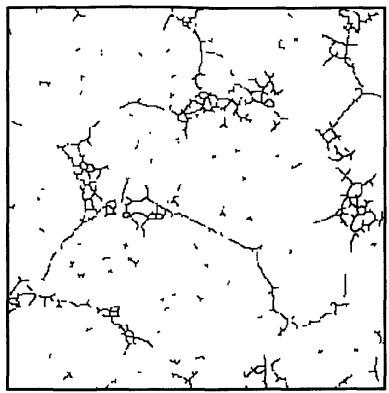

f)

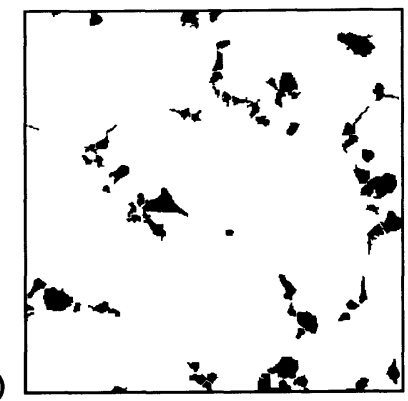

Fig. 3. - Main steps of the extraction of carbides: a) original binary image, with $\gamma$ phase in white and carbide phase in white; b) after opening of the carbides image; c) skeleton of the carbides image (the underlying light grey image reminds the opened image b); d) clipped skeleton; e) intra-dendritic carbides; f) inter-dendritic carbides after watershed. $(420 \times 420$ pixels cut from $512 \times 512$ images at mag. 1000)

as separated objects (Fig. 3f). Small filaments remain attached to some objects: their influence on the area measurement (see Sect. 4.) is small and not significant. As well as for the dendrites, the choice of the size of the transformations is interactive.

\section{Experiments and Results}

Annealing experiments were performed in a differential thermal analysis furnace $[1,3]$. The device enables a good control of the annealing temperature and duration. Backscattered electron digital images $(512 \times 512$ pixels $)$ were acquired on a Jeol JSM 6400 microscope with a Tracor TN 5500 equipment, then transferred on the TN8502 image analysis system. 10 images at magnification $\times 150$ to $\times 200$ and 16 images at magnification $\times 400$ to $\times 500$ regularly distributed on the sample sections were used for the $\gamma$ dendrites and carbides respectively. 500 to 1000 particles were counted per sample in each case. The well contrasted images were enhanced using a "min/max" rank filter before being thresholded, to reduce the human dependence of the results. Some series of measurements were realized twice, by two of the authors independently, in order to verify the reproducibility, with satisfactory results.

Surface area fractions were measured to verify that the volume fraction does not evolve with time, which is a condition for Ostwald ripening, and size measurements were needed to characterize the kinetics of the phenomenon. Individual size analysis was chosen in order to compare with Ostwald ripening models. Lemoisson et al. [4] obtained results on the inter-dendritic carbides growth using 2-D opening granulometry on a similar high speed steel. Their inter-dendritic carbides were very large, and could be associated to the second mode of the bimodal distribution. The method was tested on our alloy but did not lead to such a possibility. 

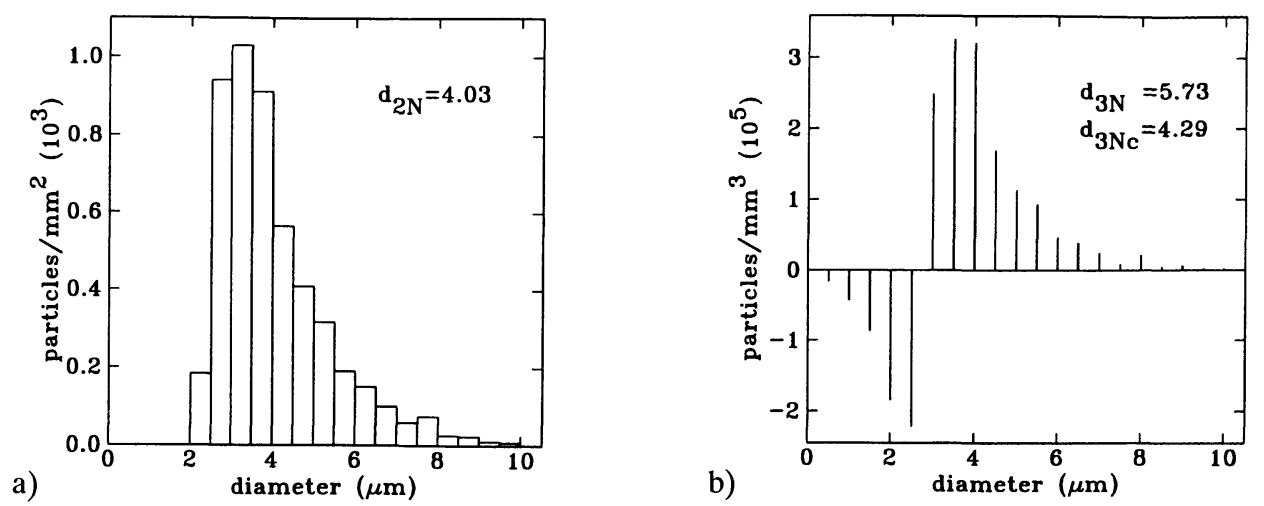

Fig. 4. - Size distribution of inter-dendritic carbides ( $\left.T_{\mathrm{e}}-13 \mathrm{~K}, 20 \mathrm{~min}\right)$ : a) 2D histogram of equivalent diameters $\left(d_{2 N}=\right.$ number weighed mean diameter); b) calculated 3D discrete (bars) equivalent distribution of spheres $\left(d_{2 N}=\right.$ crude mean diameter, $d_{2 N c}=$ negative values ignored $)$.
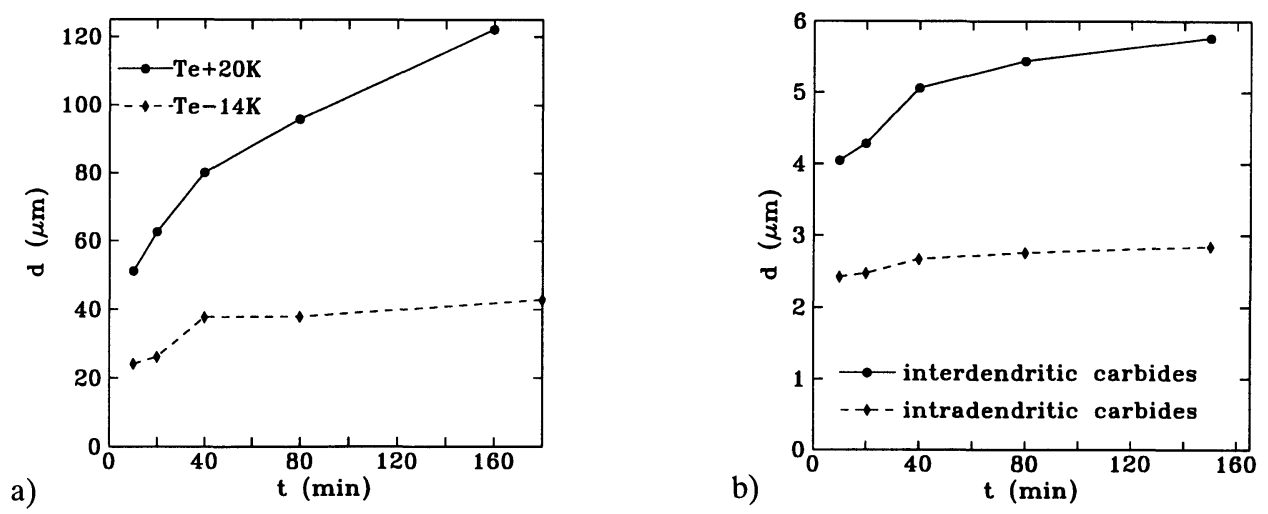

Fig. 5. - Size evolution: a) $\gamma$ dendrites below $\left(T_{\mathrm{e}}-14 \mathrm{~K}\right)$ and above $\left(T_{\mathrm{e}}+20 \mathrm{~K}\right)$ the eutectic temperature; b) intra and inter-dendritic carbides at $T_{\mathrm{e}}-13 \mathrm{~K}$.

The individual surface area $A$ of each object, the robustest size parameter, was measured. The equivalent diameter (diameter $d=\sqrt{4 A / \pi}$ of the circle with the same area) was used, as the corresponding length-dimensional parameter. The results were 3-D converted using the usual Schwartz-Saltykov method [5]. This method, based on the hypothesis of spherical particles with a discrete size distribution, has often been used for rounded objects such as our globular "dendrites".

The use for facetted $\mathrm{M}_{6} \mathrm{C}$ carbides is more questionable, and has to be considered carefully. In practice, the method does not lead to "the true" 3-D size distribution, but to the discrete 3-D size distribution of spheres which lead to the same area distribution histogram as the considered set of facetted particles. It was used within this limits, the available Ostwald ripening models [6, 7] being written with the same assumption.

In some cases (Fig. 4), some negative values appear on the left side of the size distribution of carbides, and introduce an overestimation of the mean size $d_{3 N}$. This corresponds to the lack of 2-D data for small objects, due to the morphological openings in the preceding treatment. In that particular case, the negative values can be set to zero. This provides an improvement of the measurements, which partly reintroduces the eliminated particles, and a better estimation $d_{3 N c}$ of the mean size. 
Number and volume weighed distributions and mean sizes were used, the former being suitable for comparison with models, the latter for mechanical properties and visual appreciation. A difference up to a factor of 1.5 was observed in some cases, owing to some wide size distributions.

Beyond the usual evaluation of the exponent of a power law $d^{n}=K\left(t+t_{0}\right)$, the quantitative comparison between the experimental coarsening rates and the model predictions were performed. The dendrite growth (Fig. 5a) is compatible with a diffusion controlled mechanism $(n=3)$ when carbides are not present, and slowered by the presence of carbides ("pinning effect" [8]). The intra-dendritic carbides growth (Fig. 5b) is small but larger than expected with solid state diffusion; this shows that the mechanism of $\mathrm{M}_{6} \mathrm{C}$ entrapping from the liquid by growing dendrites is significant. The inter-dendritic carbides growth rate is slower than expected: a model was developped, based on the description of diffusion inside the liquid corridors between dendrites, which explains these results (with $n=4$ ) [3,9].

\section{Conclusions}

This paper illustrates an usual stage of quantitative metallography when searching for some evolution mechanisms. Pertinent features have to be found from a complex but organized structure. The strategy first involves the analysis of the microstructure to get the guidelines for extracting significant objects. Then image analysis tools - mathematical morphology here - are combined in an adapted sequence of transformation. Finally, a choice of measurements is performed among the available parameters and methods of stereology. Questions are often encountered - use of Saltykov method for non-spherical particles, negative values in the size distribution... - and choices have to be made. In the present case, evolution data on the three phases of interest have been obtained and compared with models $[3,9]$.

\section{References}

[1] Durand-Charre M., Chaix J.M. and Yang P.H., The effect of grain growth upon eutectic carbide particle coarsening in HSS, Proc. 5th International Symposium on the Physical Metallurgy of Cast Iron, Nancy, France, 3-6 oct. 1994, J. Lacaze and G. Lesoult, Eds., Trans. Tech. Publ., in press.

[2] Coster M. and Chermant J.L., Précis d'analyse d'images (Ed. du CNRS, 1985, 1989).

[3] Yang P.H., Cinétique de coalescence des dendrites et des carbures dans des alliages de type aciers à outils, Thèse de l'INP-Grenoble (1995).

[4] Yang P.H., Chaix J.M., Durand-Charre M., Lemoisson F., Grillon F. and Bienvenu Y., Study of size evolution of $\mathrm{M}_{6} \mathrm{C}$ carbides during sintering of high speed steels by quantitative image analysis, Proc. PM’94, 1994 Powder Metallurgy World Congress, Paris, France, 6-9 june 1994, vol. III (Les Éditions de Physique, Les Ulis, 1994) pp. 2291-2294.

[5] De Hoff R.T. and Rhines F.N., Microscopie quantitative (Masson, 1972) (original ed., Quantitative Microscopy, McGraw-Hill, 1968).

[6] Voorhees P.W. and Glicksman M.E., Ostwald ripening during liquid phase sintering-effect of volume fraction on coarsening kinetics, Met. Trans. A 15A (1984) 1081-1088.

[7] Calderon H.A., Voorhees P.W., Murray J.L. and Kostorz G., Ostwald ripening in concentrated alloys, Acta Met. Mater. 42 (1994) 991-1000.

[8] Rios P.R., On the relationship between pinning force and limiting grain radius, Acta Met. Mater. 40 (1992) 873-875.

[9] Yang P.H., Durand-Charre M. and Chaix J.M., to be published. 